\title{
Aplicação de técnicas de manipulação em dados magnetométricos para interpretação de estruturas na Sub-bacia de Jandiatuba (Bacia do Solimões)
}

Rodrigo da Silva Canário*, Jonne Clay Fonseca Vidal ${ }^{2}$ e Cleverson Guizan Silva ${ }^{3}$, Observatório Nacional ${ }^{2}$, Universidade Federal Fluminense ${ }^{3}$. ${ }^{\star}$ Apresentador.

Copyright 2016, SBGf - Sociedade Brasileira de Geofísica

Este texto foi preparado para a apresentação no VII Simpósio Brasileiro de Geofísica, Ouro Preto, 25 a 27 de outubro de 2016. Seu conteúdo foi revisado pelo Comitê Técnico do VII SimBGf, mas não necessariamente representa a opinião da SBGf ou de seus associados. É proibida a reprodução total ou parcial deste material para propósitos comerciais sem prévia autorização da SBGt.

\section{Abstract}

Potential field data (magnetic) can be very useful in geological interpretation, but the complexity of the signal composition that come from several sources can often lead geophysicists to failure. For that matter this study shows the application of some techniques such as Spectral Analysis and Fourier Filters to systematically identify some of the geological sources of subsurface, allowing to estimate the depth of the structures present in the basin. It presents some preliminary results and discussions were made based on regional geological knowledge. The data used here were acquired at Jandiatuba Sub-Basin, west of Solimões Basin.

\section{Introdução}

Algumas bacias sedimentares apresentam um desafio a mais para a exploração com sísmica, já que a presença de soleiras dificulta muito o imageamento sísmico (Wanderley Filho, 2005; Sobrinho, 2007), e nesse caso os métodos potenciais têm muito a oferecer como alternativa, como por exemplo para delimitação de domos de sal através de modelagem direta ou inversa 3D em dados potenciais (Silva et al., 2011). Por isso este trabalho utilizou técnicas (Transformada de Fourier, Análise Espectral e Processos de Filtragem) aplicadas ao processamento de dados magnetométricos no auxílio à interpretação preliminar da geologia integrando informações de poços e de linhas sísmicas. Os dados utilizados no trabalho foram obtidos através da ANP pelo projeto 0001_SOLIMÕES_JANDIATUBA realizado na Sub-Bacia de Jandiatuba, na porção oeste da Bacia do Solimões conforme mostra a figura 1.

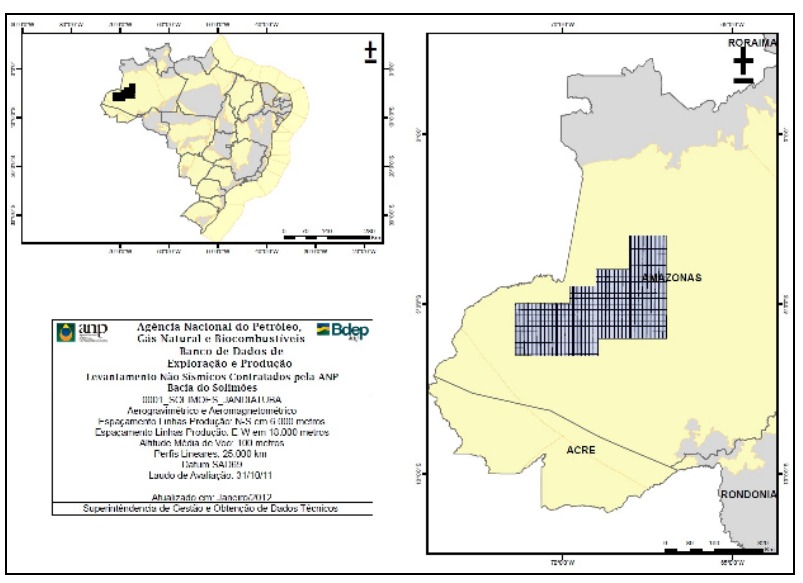

Figura 1 - Localização da área de aquisição do aerolevantemento referente ao projeto 0001_SOLIMÕES_JANDIATUBA fornecidos pela ANP.

A Bacia do Solimões é uma bacia de depressão paleozóica intracratônica e está situada no estado do Amazonas (figura 2), onde ocupa uma área de $600.000 \mathrm{~km}^{2}$ dos quais $480.00 \mathrm{~km}^{2}$ são propícios à prospecção de hidrocarbonetos (Eiras,1994; Eiras, 2006). Ela é limitada ao norte pelo Escudo das Guianas e ao sul pelo Escudo Brasileiro, a leste pelo Arco de Purus e a oeste pelo Arco de Iquito (Milani, 1991). Internamente a bacia é compartimentada pelo Arco de Carauari (Porshe, 1985), que a segmenta em duas sub-bacias, a sub-Bacia de Jandiatuba a oeste e a sub-Bacia de Juruá a leste (figura 3).

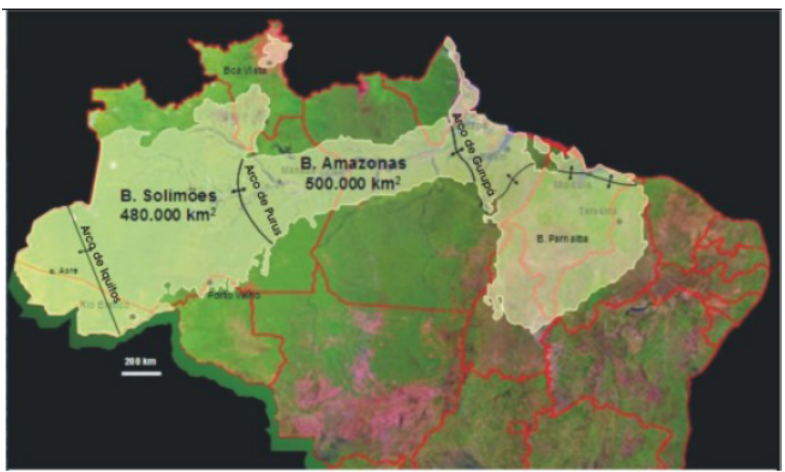

Figura 2 - Localização da Bacia do Solimões, destacando os arcos que a divide com a Bacia do Amazonas e com a Bacia do Acre. Fonte: Adaptado de Wanderley Filho (2005). 


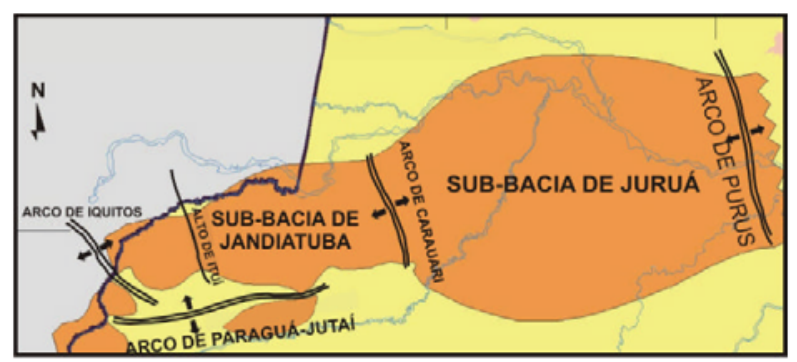

Figura 3 - Divisão da Bacia do Solimões a partir do Arco de Carauarí, a oeste Sub-Bacia de Jandiatuba e a lesta, Sub-Bacia de Juruá (Fonte: Clark, 2002).

Segundo Wanderley Filho et al. (2007) a Bacia do Solimões sob critérios litoestratigráficos pode ser definida em cinco sequências deposicionais:

- $\quad F m$. Benjamin Constant (Ordoviciano)

- Fm Jutaí (Siluriano Superior-Devoniano Inferior)

- Grupo Marimari (Devoniano Médio-Carbonífero Inferior)

- Grupo Tefé (Carbonífero Superior-Permiano)

- Grupo Javari (Cretáceo Superior-Quaternário

Inclui-se ainda um evento magmático ocorrido por volta de 204 Ma (Triássico) que coincide com o Magmatismo Penatecaua (Eiras e Wanderley Filho, 2006). Esse evento magmático foi responsável pelas intrusões em forma de soleira que são identificadas hoje em linhas sísmicas, na maior parte da bacia são identificadas pelo menos três soleiras, todas deformadas por um evento tectônico no Mesozóico conhecido como Tectônica do Juruá. Esse tectonismo transpressivo reativou antigas estruturas, gerando falhas reversas de alto ângulo com anticlinais associados (figura 4) (Caputo, 2014; Barata \& Caputo, 2007).

Nesse contexto é que se caracterizou um grande movimento destrógiro (ENE-WSW) que atravessa quase toda a bacia, denominado Megacisalhamento Solimões (Caputo e Silva, 1990) e possui 5 trends associados em escalonamento (figura 5).

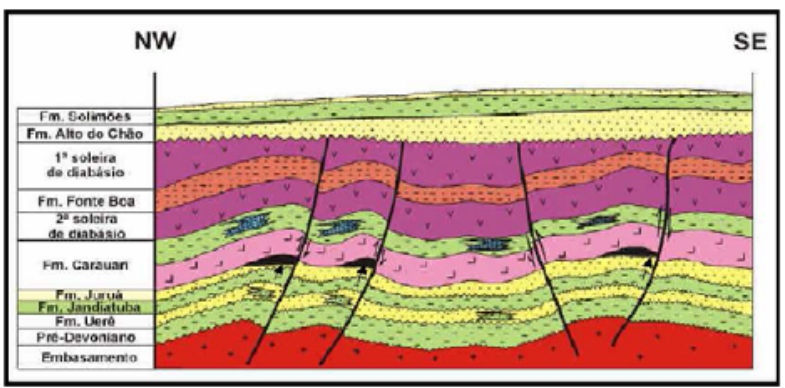

Figura 4 - Falhas de alto ângulo associadas a dobras anticlinais representando elementos do sistema (Eiras, 1998).

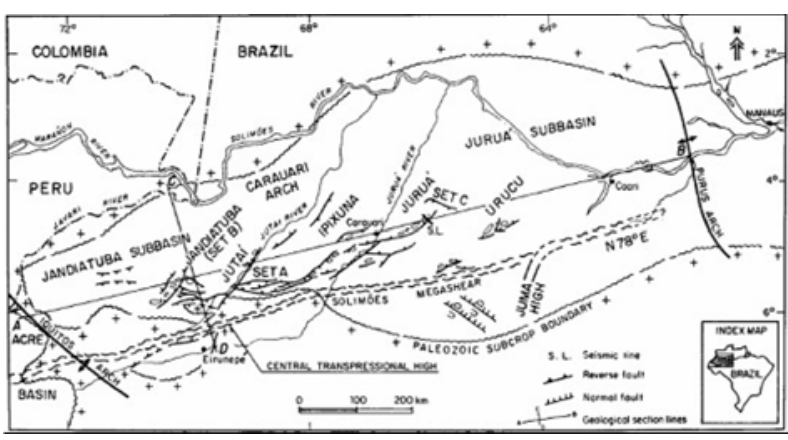

Figura 5- O Megacisalhamento Solimões identificado por linhas tracejadas e trends estruturais escalonados por linhas pontilhadas (Fonte: ANP).

\section{Metodologia}

A metodologia utilizada neste trabalho concerne análise espectral e alguns filtros em concomitância para a interpretação estrutural preliminar de estruturas geológicas. Algumas das técnicas bem estabelecidas para a interpretação de gravimetria e magnetometria são:

- $\quad$ Espectro de Potência (Spector e Grant, 1970)

- Filtragem de Fourier 2D (Gupta e Grant, 1985)

- Métodos automatizados de estimativa de profundidade de fontes (Barbosa et al., 2005)

Primeiro foi feita a transformação dos dados potencias do domínio do espaço para o domínio da frequência através da FFT(Fast Fourier Transform), o que permite a aplicação de diversos filtros para realçar determinadas feições geológicas de interesse para o estudo.

Antes da aplicação desses filtros foi analisado o espectro de potência radial médio dos dados potenciais segundo a técnica proposta por Spector e Grant, 1970, que coloca o logarítmo da energia do espectro de potencia de um campo potencial em função do número de ondas associado a uma distribuição de fontes em subsuperfície.

Através da análise dos coeficientes de inclinação das retas (figura 6) ajustadas ao espectro de potência do 
conjunto de dados é possível estimar uma profundidade para o topo dos corpos causativos.

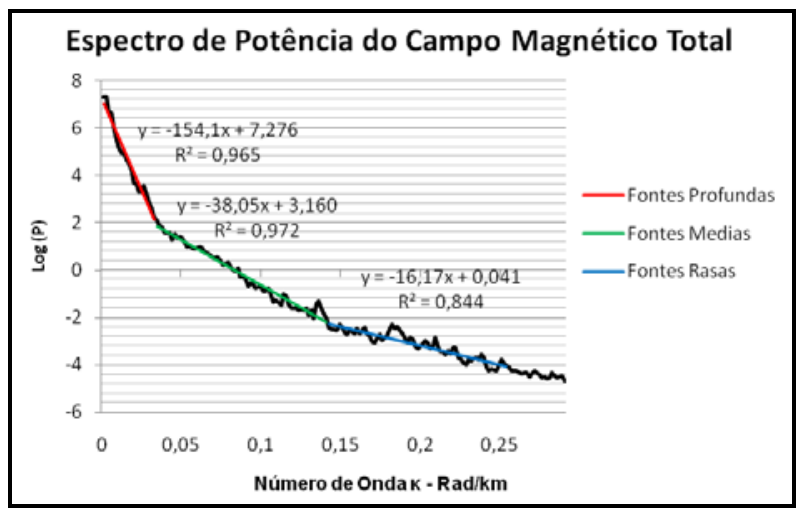

Figura 6 - Gráfico de espectro de potencia (log) contra o número de onda $(\mathrm{rad} / \mathrm{km}) \mathrm{com}$ as retas ajustadas e seus respectivos coeficientes.

Através da fórmula $\mathrm{H}=-\mathrm{S} / 4 \pi$ (Spector e Grant, 1970) a profundidade $\mathrm{H}$ foi calculada a partir da inclinação $\mathrm{S}$ das respectivas retas ajustadas conforme mostra a tabela 1.

A tabela 1 abaixo dá uma descrição simplificada dos filtros que operam no domínio de Fourier 2D.

Tabela 1 - Filtros no domínio de Fourier e suas características

\begin{tabular}{|c|c|}
\hline Filtro & Características \\
\hline $\begin{array}{c}\text { Amplitude do Sinal } \\
\text { Analítico }\end{array}$ & $\begin{array}{c}\text { Intrusivas (diques e } \\
\text { soleiras), feições } \\
\text { estruturais. }\end{array}$ \\
\hline Derivadas Direcionais & $\begin{array}{c}\text { Falhas, contatos e centro } \\
\text { de intrusivas verticais }\end{array}$ \\
\hline
\end{tabular}

Derivadas direcionais - Utilizando tanto o Campo Magnético Total quanto a anomalia isostática gravimétrica foi aplicado o filtro de derivada, onde trends, zonas de falha e contatos litológicos são evidenciados pelos valores máximos e mínimos apresentados no processo de derivação.

\section{Resultados}

Através da fórmula $\mathrm{H}=-\mathrm{S} / 4 \pi$ (Spector e Grant, 1970) a profundidade $(\mathrm{H})$ foi calculada a partir da inclinação(S) das respectivas retas ajustadas conforme mostra a tabela 2.
Tabela 2 - Tabela de Parâmetros obtidos na Análise Espectral

\begin{tabular}{|c|c|c|}
\hline \multicolumn{3}{|c|}{ MAGNETOMETRIA } \\
\cline { 1 - 2 } $\begin{array}{c}\mathrm{N}^{0} \text { de Onda } \\
\text { (rad/km) }\end{array}$ & $2.53 \times 10^{-1}$ & \multirow{2}{*}{ Fontes Rasas } \\
\cline { 1 - 2 } $\begin{array}{c}\text { Profundidade } \\
\text { (km) }\end{array}$ & 1.28 & \multirow{2}{*}{ Fontes Médias } \\
\cline { 1 - 2 } $\begin{array}{c}\text { No de Onda } \\
\text { (rad/km) }\end{array}$ & $1.41 \times 10^{-1}$ & \\
\hline $\begin{array}{c}\text { Profundidade } \\
\text { (km) }\end{array}$ & 3.02 & \multirow{2}{*}{ Fontes Profundas } \\
\cline { 1 - 2 } $\begin{array}{c}\text { No de Onda } \\
\text { (rad/km) }\end{array}$ & $3.33 \times 10^{-2}$ & \\
\hline $\begin{array}{c}\text { Profundidade } \\
\text { (km) }\end{array}$ & 12.26 & \\
\hline
\end{tabular}

Através da análise espectral foi possível definir três níveis de profundidade para as fontes: fontes rasas, fontes médias e fontes profundas.

As fontes rasas podem estar correlacionadas com rochas intrusivas, como diques e soleiras de diabásio, amplamente distribuídas pela Bacia do Solimões. As fontes médias provavelmente são provenientes do embasamento cristalino, que na Sub-Bacia de Jandiatuba fica em torno de $3 \mathrm{~km}$ a $3.4 \mathrm{~km}$. As fontes profundas são de difícil interpretação, mas no contexto interpretativo apresentado pelo trabalho possivelmente tratam-se de dutos que alimentaram as soleiras.

Em paralelo à análise espectral foram confeccionados os mapas para a intepretação geofísica/geológica, procurando lineamentos estruturais e outras estruturas.

Esses mapas foram obtidos a partir do Campo Magnético Total (TMI), mostrado na figura 7, e a partir dele foram gerados todos os outros mapas baseados em filtros no domínio de Fourier. 


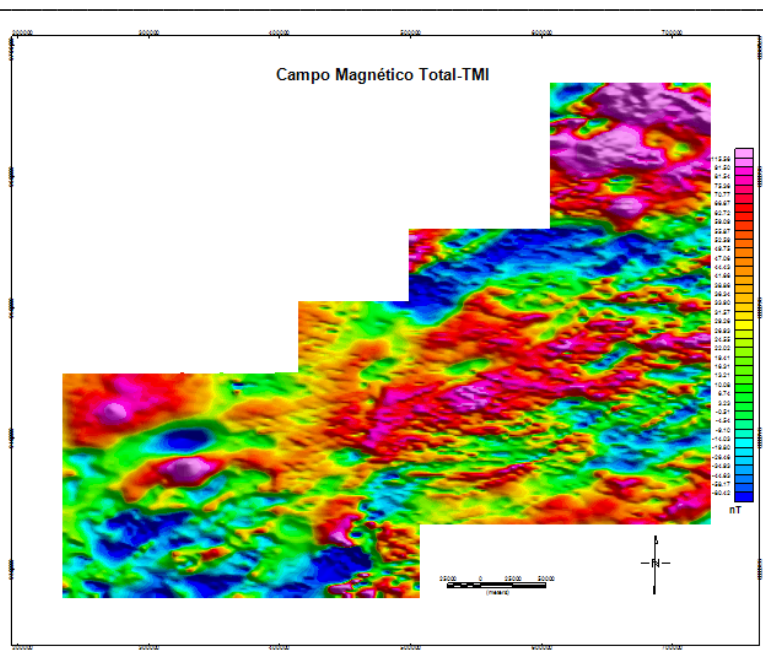

Figura 7 - Campo Magnético Total.

O mapa mostrado na figura 8 é a primeira derivada vertical, que tende a destacar fontes mais rasas, e nele foram intepretadas duas províncias magnéticas delimitadas pelas linhas brancas, uma a leste e outra a oeste, podendo estar associadas a duas grandes zonas de intrusões. Na província a oeste nota-se a presença de um dipolo magnético bem caracterizado, identificado por uma circunferência em preto, o mesmo é também é observado na figura 7. Alguns lineamentos de direção NE-SO também são mostrados em preto, eles coincidem com os trends estruturais da bacia. O lineamento magnético marcado ao sul, em vermelho, pode estar associado à zona transpressiva do Megacisalhamento Solimões (figura 5), a localização geográfica é praticamente coincidente.
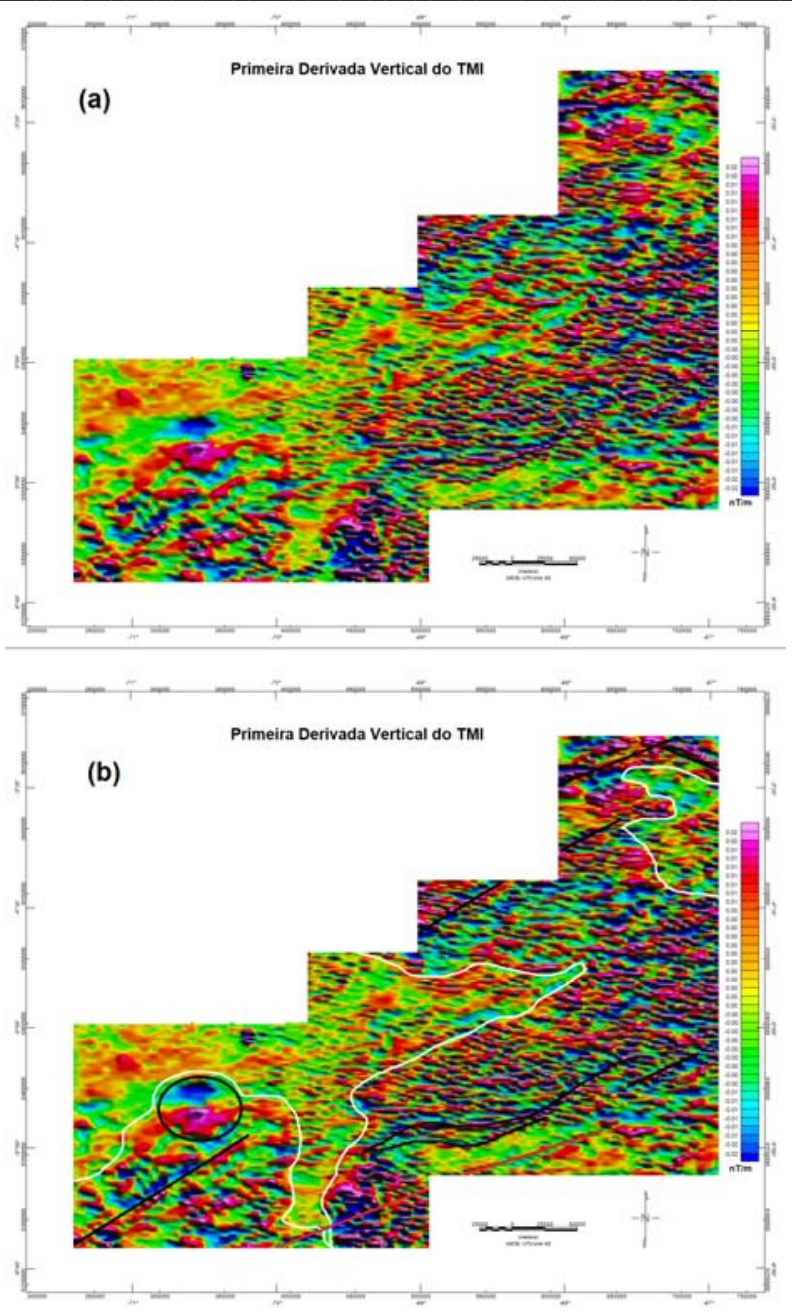

Figura 8 - $1^{\circ}$ Derivada Vertical (a)Sem interpretação, (b) Com interpretação .

A figura 9 apresenta o mapa da Amplitude do Sinal Analítico, esse filtro tende a mapear melhor as bordas dos corpos, particularmente me fontes rasas, e por isso a província marcada a oeste pôde ser delimitada com maior detalhe. Nesse mapa foram avistados quase todos os lineamentos do mapa na figura 8, confirmando a interpretação prévia. 

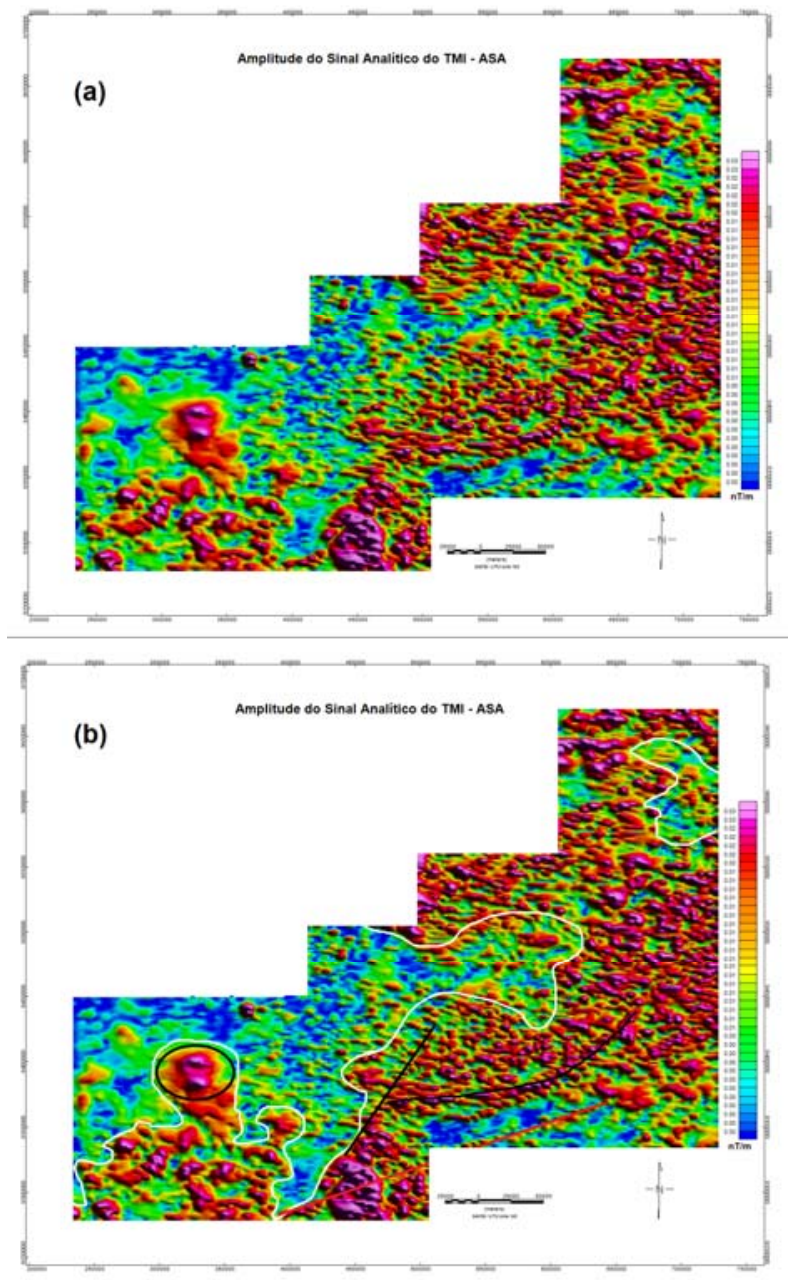

Figura 9 - Amplitude do Sinal Analítico

Na figura 10 é mostrado o mapa do Gradiente Horizontal Total, usado para avaliar mudanças lateralmente abruptas nas propriedades físicas que ocorrem em subsuperfície. Nesse mapa não ficou tão clara a definição das duas províncias anteriormente mapeadas, mas foi possível marcar alguns outros lineamentos de orientação NE-SO que não apareciam nos outros mapas.

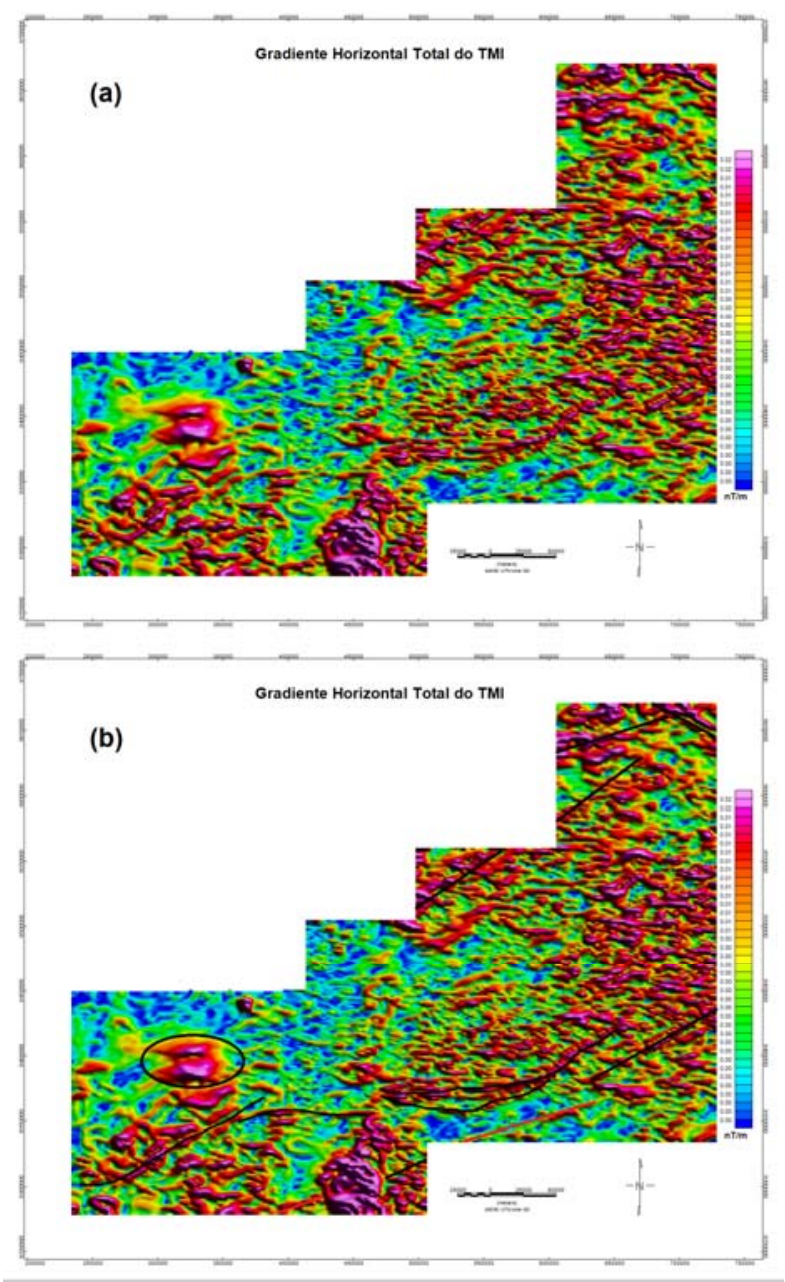

Figura 10 - Gradiente Horizontal Total do TMI

\section{Discussão e Conclusões}

O conteúdo apresentado neste trabalho mostra como a aplicação de técnicas aplicadas em dados magnéticos podem auxiliar no entendimento geológico de uma bacia sedimentar. Contudo, os resultados apresentados aqui demanda maiores exames, especialmente com maior integração com dados sísmicos e poços. Esses resultados representam uma prévia dos principais "trends" geológicos da bacia, de maneira que a ambiguidade presente nos dados magnéticos precisa ser considerada. A natureza dipolar dos dados magnéticos não permite uma delimitação inequívoca dos corpos causativos. Além disso, uma grande incógnita que se coloca a todo levantamente magnetométrico é se a magnetização dos corpos é remanente. Dessa forma, as fontes profundas colocadas na tabela 2 não necessariamente significam variações intra-crustais, mas podem corresponder a uma maior expressão volumétrica das intrusões, ou ainda de uma maior proximidade destas com a superfície. 


\section{Agradecimentos}

Os autores deste trabalho agradecem ao LAGEMAR, ao DOT e à UFF por terem fornecido recursos de software e infraestrutura para a execução. Agradecem também à ANP, que forneceu os dados utilizados.

\section{Referências}

Almeida, F. F. M., 1969. Diferenciação tectônica da Plataforma Brasileira (Tectonic differentiation of the Brazilian Platform). Proceedings 33rd. Brazilian Geological Congress, Salvador (BA), Brazil, vol. 1: 29-46.

Barata, C.F. , Caputo, M. V. - 2007 - Geologia do Petróleo da Bacia do Solimões. o "Estado da Arte".

Barbosa, V.C., Batista, J.C., 2005. Deconvolução de Euler: passado, presente e futuro - um tutorial. Revista Brasileira de Geofísica, Vol. 23 (3), pg. 243-250.

Caputo M. \& Silva O.B. 1990. Sedimentação e Tectônica da Bacia do Solimões. In: Raja Gabaglia G.P. \& Milani E.J. (coords.) Origem e Evolução de Bacias Sedimentares. Petrobras, $415 \mathrm{p}$.

Caputo, Mario Vicente. Juruá Orogeny: Brazil and Andean Countries. Braz. J. Geol., São Paulo, v. 44, n. 2, p. 181-190, June 2014.

Clark, J. 2002. Bacia do Solimões. Quarta Rodada de Licitação - Superintendência de Definição de Blocos. Seminário Jurídico/Fiscal e Workshop Técnico da Quarta Rodada de Licitações. Petrobrás. 38p

Cordani, U.G. et al., 1983. Estudo preliminar de integração do Pré-Cambriano com os eventos tectônicos das bacias sedumentares brasileiras. Rio de Janeiro. PETROBRAS/CENTPES, 70 p. (Série Ciência-TécnicaPetróleo. Seção: Exploração de Petróleo no. 15).

Eiras, J. F., Becker, C. R., Souza, E. M., Gonzaga, F. G., Silva J. G. F., Daniel, L. M. F., Matsuda, N. S., Feijó, F. J., 1994. Bacia do Solimões. Boletim de Geociências da Petrobras, Rio de Janeiro: CENPES, v. 8, n. 1, p. 17-45, jan./março.

Eiras, J.F. 1998. Tectônica, sedimentação e sistemas petrolíferos da Bacia do Solimões, Estado do Amazonas. Searching For Oil and Gas in the Land of Giants. The Search. Rio de Janeiro, edição especial sobre o Brasil, cap. 2, p.23-31.

Eiras, J.F.; Wanderley Filho, J.R., 2006. Bacias Sedimentares Brasileiras (Bacia do Solimões) Fundação Paleontológica Phoenix.

Gabaglia, G. P. R. \& Milani, E. J., cords. 1990. Origem e evolução de bacias sedimentares. Rio de Janeiro, Petrobras, 415p.

Gupta, V.K., Grant, F.S., 1985. Mineral-exploration aspects of gravity and aeromagnetic surveys in the Sudbury-Cobalt area, Ontario; in The Utility of Regional Gravity and Aeromagnetic Anomaly Maps, Society of Exploration Geophysicists, Special Volume, p.392-411.
Munis, M.B. 2009. Caracterização Geomagnética do Gráben Purus e suas implicações na evolução das bacias do Solimões e Amazonas. Tese de Doutoramento, UFRJ/COPPE, 2009. XII, 102p.

Silva Dias, F.J.S., Barbosa, V.C.F., Silva, J.B.C., Vasconcelos, S.S., Oliveira, F.S., 2011. Adaptive learning 3D gravity inversion for salt-body imaging. Geophysics, v. 76, no. 3, p. 149/157.

Sobrinho, M. K. M., 2007. Respostas MT de Soleiras de Diabásio da Província Petrolífera de São Mateus (Solimões-AM).

Spector, A., Grant, F.S., 1970, Statistical models for interpreting aeromagnetic data. Geophysics, Vol 35, No.2, p. 293-302.

Porsche, E. 1985. Tectônica da faixa de dobramentos do Juruá. Bacia do Alto Amazonas. Brasil: um modelo estrutural. 214 p. Tese (Mestrado) - Universidade Federal de Ouro Preto, Ouro Preto, 1985.

Wanderley Filho, J. R.; Travassos, W. A. S.; Alves, D. B. O diabásio nas bacias paleozóicas amazônicas - herói ou vilão. Boletim de Geociências Petrobras, Rio de Janeiro: CENPES, v. 14, n. 1. p. 117-184, nove. 2005/ maio 2006.

Wanderley Filho, J. R. W.; Eiras, J.F.; Vaz, P.T. 2007. Bacia do Solimões. Boletim de Geociências Petrobras, Rio de Janeiro, v. 15, n.2, p.217-225, maio/nov. 